\title{
Clinical Features of Idiopathic Nephrotic Syndrome and Factors Associated with its Relapse in Children: A Population-Based Study
}

\author{
Abolhassan Seyezadeh ${ }^{1}$, Mohamad Reza Tohidi ${ }^{1,}{ }^{*}$, Somaye Sheikhi ${ }^{1}$, Mohammad Saleh Seyedzadeh ${ }^{1}$ \\ and Sara Hookari ${ }^{1}$ \\ ${ }^{1}$ Department of Pediatrics, Pediatric Nephrology Division, Kermanshah University of Medical Sciences, Kermanshah, Iran \\ "Corresponding author: Department of Pediatrics, Pediatric Nephrology Division, Kermanshah University of Medical Sciences, Kermanshah, Iran. Email: \\ tohidimohamadreza63@gmail.com
}

Received 2020 August 04; Revised 2020 October 03; Accepted 2020 November 22.

\begin{abstract}
Background: Idiopathic Nephrotic Syndrome (INS), which is caused by a defect in the glomerular filtration barrier, is the most common chronic glomerular disease in children.

Objectives: The present study aimed to assess the clinical features of INS and some recurrence-related factors in children.

Methods: This population-based, cross-sectional study was conducted on 302 children with INS referring to the Pediatric Nephrology Clinic of Imam Reza Hospital of Kermanshah city, Iran, during 1998-2018.

Results: The mean age (SD) at the time of diagnosis and the follow-up duration were 4.87 (2.89) years and 49.83 (37.52) months, respectively. The numbers of boys and girls were 185 (61.9\%) and 114 (38.1\%), respectively. The mean number (SD) of recurrences, annual recurrence rate during the follow-up, and the time to the first recurrence after responding to treatment were 1.71 (1.91), 0.48 (0.77), and 10.15 (10.63) months, respectively. The most common type of INS was steroid-dependent/frequent relapse, with a frequency of 151 (50.5\%). Furthermore, 33 (11.0\%) and 266 (89.0\%) patients were resistant and respondent to treatment, respectively. There was a statistically significant relationship between age at the time of diagnosis and the type of INS ( $\mathrm{P}=0.007)$.

Conclusions: This study revealed a statistically significant association between higher age and steroid resistance. However, in steroid responders, there was no relationship between gender, age at the time of diagnosis, and the time to the first recurrence, and the recurrence rate.
\end{abstract}

Keywords: Idiopathic Nephrotic Syndrome, Relapse, Cross-Sectional Study, Children, Iran

\section{Background}

Idiopathic Nephrotic Syndrome(INS), as the most common chronic glomerular disease in children, is characterized by protein excretion $>40 \mathrm{mg} / \mathrm{m}^{2} / \mathrm{h}$ or $>50 \mathrm{mg} / \mathrm{kg} /$ day, hypoalbuminemia $\leq 2.5 \mathrm{~g} / \mathrm{dl}$, edema, and hyperlipidemia $(1,2)$. This syndrome is caused by damage to the glomerular basement membrane of the kidney that increases the permeability of the Glomerular Filtration Barrier (GFB) and causes the kidney to excrete a large number of proteins $(3,4)$. The annual incidence of INS is $2-3$ per 100,000 children in most western countries. However, the incidence rate is higher in developing and underdeveloped countries $(5,6)$. Etiologically, nephrotic syndrome is divided into two types, including primary (idiopathic) and secondary (after systemic diseases). About 95\% of cases are idiopathic in pediatrics. Idiopathic nephrotic syndrome is more prevalent in boys, and the most common age of onset is 2-6 years.
The most common pathologic features of INS are minimal change disease (85\%), meningeal proliferation (5\%), and focal segmental glomerulosclerosis (10\%). Secondary nephrotic syndrome may be related to glomerular disease or systemic diseases such as lupus erythematosus $(2,5,7)$.

Corticosteroids are the mainstay for the treatment of INS. Approximately $90 \%$ of children with INS respond appropriately to treatment, which is an important factor in predicting the course of the disease. However, the high incidence of relapse and resistance to treatment leads to the complexity of treatment and makes parents concern about the prognosis of the disease and the future of their children. Some studies have shown that at least $50 \%$ of these children can be defined as steroid-dependent or frequent relapser (8-10). About 40-70\% of children experience recurrence once or several times after eliciting a good response to treatment. Recurrent relapse increases the risk of side effects of corticosteroids and immunosuppressive therapy 
in these children $(11,12)$. Therefore, the recurrence is one of the most important problems in the care of children with INS, although there is restricted success in correlating the course of nephrotic syndrome in children and the laboratory and clinical characteristics at the onset of disease. Some studies have found that factors such as the number of relapses within the first six months, low age of patients, low birth weight, no response to corticosteroids in the first month of initial treatment, and some other factors such as a higher level of lipoproteins at the onset are effective in predicting further relapses (9,13-15).

\section{Objectives}

According to the above explanations and the limited number of studies conducted on the clinical course of the disease, the factors related to prognosis, and the ways of responding to treatment in patients with INS, the current research intended to assess the clinical features of INS and some relevant factors associated with its relapse in children referring to the Pediatric Nephrology Clinic of Imam Reza Hospital of Kermanshah city during 1998-2018.

\section{Methods}

This research was a population-based cross-sectional study conducted on 299 children with INS referring to the Pediatric Nephrology Clinic of Imam Reza Hospital of Kermanshah city, Iran, during 1998-2018. In the present study, the diagnosis of INS in children was based on nephrotic range proteinuria ( $>40 \mathrm{mg} / \mathrm{m}^{2} / \mathrm{h}$ ), hypoalbuminemia, hypercholesterolemia, and edema. All patients were treated with $60 \mathrm{mg} / \mathrm{m}^{2} /$ day oral prednisolone for four weeks, followed by $40 \mathrm{mg} / \mathrm{m}^{2} /$ day for the other four weeks; thereafter, the dose gradually decreased and discontinued in three to six months. A kidney biopsy was performed on patients who showed resistance to treatment, and proper treatment was assigned based on the pathological findings. We also used drugs such as levamisole, cyclosporine, mycophenolate mofetil, and rituximab that has been used in recent years as adjuvant therapy for frequent relapsers or steroid-dependent patients. The inclusion criterion included all patients diagnosed with INS. The exclusion criteria were secondary or non-idiopathic nephrotic syndrome, Congenital Nephrotic Syndrome (CNS), and incomplete medical records.

Given that the population under study included all children with INS referring to the Pediatric Nephrology Clinic of Imam Reza Hospital of Kermanshah city, Iran, during 1998-2018, the study used a census method, and it was not required to calculate the sample size. The data collection tool was a checklist including demographic variables including age and sex and clinical variables including follow-up, number of recurrences, annual recurrence rate, time to first recurrence after responding to treatment, type of nephrotic syndrome, type of drug used, type of response to treatment, kidney biopsy results, side effects of INS (infection, high blood pressure, acute renal failure, anemia, thromboembolism, and leukopenia), and side effects of medications (cataract, cushingoid face, high blood pressure, leukopenia, increased liver enzymes, and renal impairment). All of these variables were extracted from patients' medical records.

\subsection{Definitions}

Steroid Resistant: No remission after four weeks of daily prednisone treatment with a dose of $60 \mathrm{mg} / \mathrm{m}^{2}$, followed by three methylprednisolone pulses (16).

Frequent Relapser: Two or more relapses within six months of the initial response, or four or more relapses within one year (16).

Steroid Dependent: Two consecutive relapses during prednisolone therapy or within 14 days after the cessation of prednisolone (16).

\subsection{Statistical Analysis}

Data were analyzed using SPSS V. 22 software and descriptive statistics. After determining the prevalence of some factors associated with relapse, the chi-square test was used to evaluate the study hypotheses. The P values of less than 0.05 were considered statistically significant.

\section{Results}

The present population-based, cross-sectional study was conducted on 302 children with INS referring to the Pediatric Nephrology Clinic of Imam Reza Hospital of Kermanshah city, Iran, during 1998-2018. Table 1 shows the quantitative descriptive variables of pediatric patients under study. As can be seen, the mean age (SD) at the time of diagnosis and the follow-up duration were 4.87 (2.89) years and 49.83 (37.52) months, respectively. Further, the mean number of recurrence, annual recurrence rates during the follow-up, and the time to the first recurrence after responding to treatment in terms of months were 1.71(1.91), 0.48 (0.77), and 10.15 (10.63), respectively (Table 1 ).

Table 2 shows the qualitative descriptive variables of children under study. The numbers of boys and girls were 185 (61.9\%) and 114 (38.1\%), respectively. The most common type of INS was steroid dependence/frequent relapse with 


\begin{tabular}{|c|c|c|c|c|}
\hline Variable & Mean & S.D $\mathrm{D}^{\mathrm{a}}$ & Minimum & Maximum \\
\hline Age at presentation (years) & 4.87 & 2.89 & 1 & 14 \\
\hline Duration of follow-up (months) & 49.83 & 37.52 & 2 & 204 \\
\hline Number of recurrences during the follow-up & 1.71 & 1.91 & 0 & 13 \\
\hline Time to the first recurrence after response to treatment (months) & 10.15 & 10.63 & 1 & 84 \\
\hline
\end{tabular}

${ }^{a}$ S.D: Standard Deviation.

a frequency of 151 (50.5\%). In terms of response to treatment, 33 (11.0\%) and 266 (89.0\%) patients were resistant and responsive to treatment, respectively. In addition, a biopsy was not performed for 264 (88.3\%) patients due to a lack of need, while all of the patients responded to treatment. The renal biopsy results showed a minimal change (4.7\%), mesangial proliferation (2.7\%), and focal segmental glomerulosclerosis (2.3\%). Other details can be seen in Table 2 .

Table 3 demonstrates the side effects of INS and medications in the patients under study. As can be seen, the most common side effects of the nephrotic syndrome were high blood pressure, infection (urinary tract infection most commonly), and anemia with $9.4,5.7$, and $1.3 \%$, respectively. Additionally, the most common drug side effects in the population under study were cataract, cushingoid face, and renal impairment with 7.7, 2.7, and 2.3\%, respectively. Other descriptions can be seen in Table 3.

Table 4 shows the relationship between the patients' age at the time of diagnosis (years) and the variables, including the type of nephrotic syndrome, number of recurrences, and annual recurrence rate during the follow-up period. As can be seen, the Chi-square test results indicated a statistically significant relationship only between age and type of nephrotic syndrome, so that resistance to treatment was more common among older children at the time of diagnosis $(\mathrm{P}=0.007)$.

Table 5 shows the relationship of the patients' sex and duration of follow-up with nephrotic syndrome typology, number of recurrences, and annual recurrence rate during the follow-up. The Chi-square test results indicated no significant statistical relationship between the patients' sex and the type of INS, number of recurrences, and annual recurrence rate during the follow-up (all P values $>0.05$ ).

\section{Discussion}

The current study aimed to assess the clinical features of INS and some factors associated with its relapse in children with nephrotic syndrome, referring to the Pediatric
Nephrology Clinic of Imam Reza Hospital of Kermanshah city during 1998-2018. The results in children who underwent a biopsy were a minimal change (4.7\%), mesangial proliferation (2.7\%), and glomerulosclerosis (2.3\%). The most common type of INS was a recurrent or dependent type, with a frequency of 151(50.5\%). Furthermore, 33 (11.0\%) and 266 (89.0\%) patients showed resistance and response to treatment, respectively. The inferential analysis demonstrated that there was a statistically significant relationship between age and the type of nephrotic syndrome ( $P$ $=0.007$ ).

Our findings are consistent with those obtained from other research carried out in Iran. For example, in the research performed by Ahmadzadeh et al., of 231 children with INS, $87 \%$ and $13 \%$ were sensitive and resistant to steroids, respectively. Besides, $38 \%$ of the patients with steroid-sensitive nephrotic syndrome were not relapsers, and 26.4 and $34.8 \%$ of them were steroid-dependent and frequent relapsers, respectively. Of 30 (13\%) resistant nephrotic syndrome patients, a renal biopsy was done in 26 patients, in which focal segmental glomerulosclerosis was the most frequent finding (17). In Wong's study, the INS incidence was 1.9 per 100,000 children younger than 15 years of age. There was no significant difference in INS between the racial groups. In addition, $80.4 \%$ were sensitive to steroids, with a mean response time of 8.4 days and a mean recurrence time of $15.10 \pm 12.10$ weeks (18). A 12-year cross-sectional study by Seyedzadeh et al., seeking the clinical features of 104 children with INS, showed that the mean age of patients (SD) was 5.57 (3.9) with the range of 1-16 years. The numbers of boys and girls were $63(60.50 \%)$ and 41 (39.50\%), respectively. The numbers of steroid-resistant and steroid-responsive patients were 26 (25\%) and 78 (75\%), respectively. Among the respondents, 39 (50\%) were frequent relapser/steroid-dependent. Besides, 19 patients underwent needle renal biopsy. Pathologic examination revealed that minimal change, focal segmental sclerosis, mesangial proliferation, and other pathologic findings were 9 (8.65\%), 5 (4.81\%), 2 (1.92\%), and 2 (1.92\%), respectively. Finally, the study concluded that most INS pa- 
Table 2. Qualitative Descriptive Variables in Patients under Study

\begin{tabular}{|c|c|}
\hline Variable & Number (\%) \\
\hline \multicolumn{2}{|l|}{ Sex } \\
\hline Boy & $185(61.9)$ \\
\hline Girl & $114(38.1)$ \\
\hline \multicolumn{2}{|l|}{ Age at presentation (years) } \\
\hline $1-2$ & $40(13.4)$ \\
\hline $2-4$ & $120(40.1)$ \\
\hline $4-6$ & $68(22.7)$ \\
\hline $6-8$ & $32(10.7)$ \\
\hline$>8$ & $39(13.0)$ \\
\hline \multicolumn{2}{|l|}{ Duration of follow-up (months) } \\
\hline$\leq 6$ & $29(9.7)$ \\
\hline $7-24$ & $63(21.1)$ \\
\hline $25-48$ & $67(22.4)$ \\
\hline $49-72$ & $67(22.4)$ \\
\hline 73-96 & $50(16.7)$ \\
\hline$>96$ & $23(7.7)$ \\
\hline \multicolumn{2}{|c|}{ Time to the first recurrence after response to treatment (months) } \\
\hline No recurrence & $82(30.8)$ \\
\hline $0-6$ & $78(29.3)$ \\
\hline $7-12$ & $62(23.3)$ \\
\hline $13-24$ & $29(10.9)$ \\
\hline $25-36$ & $8(3.0)$ \\
\hline $37-48$ & $5(1.9)$ \\
\hline$>48$ & $2(0.8)$ \\
\hline \multicolumn{2}{|l|}{ Number of recurrences during the follow-up } \\
\hline No recurrence & $82(30.8)$ \\
\hline 1 & $67(25.2)$ \\
\hline 2 & $53(19.9)$ \\
\hline 3 & $27(10.2)$ \\
\hline 4 & $18(6.8)$ \\
\hline$\geq 5$ & $19(7.1)$ \\
\hline \multicolumn{2}{|l|}{ Annual recurrence rate during the follow-up } \\
\hline None & $82(30.8)$ \\
\hline $0.01-1.00$ & $154(57.9)$ \\
\hline $1.01-2$ & $25(9.4)$ \\
\hline$\geq 2$ & $5(1.9)$ \\
\hline \multicolumn{2}{|l|}{ Type of nephrotic Syndrome } \\
\hline Resistant to treatment & $33(11.0)$ \\
\hline Steroid dependent/frequent relapse & $151(50.5)$ \\
\hline Infrequent recurrence & $33(11.0)$ \\
\hline No recurrence & $82(27.4)$ \\
\hline \multicolumn{2}{|l|}{ Type of response to treatment } \\
\hline Resistant to treatment & $33(11.0)_{-}$ \\
\hline Respondent to treatment & $266(89.0)$ \\
\hline \multicolumn{2}{|l|}{ Result of kidney biopsy } \\
\hline Disease with minimal changes & $14(4.7)$ \\
\hline Mesangial proliferation & $8(2.7)$ \\
\hline Focal segmental glomerulosclerosis & $7(2.3)$ \\
\hline Failure to perform a biopsy & $6(2.00)$ \\
\hline No need for biopsy & $264(88.3)$ \\
\hline
\end{tabular}




\begin{tabular}{lc}
\hline Table 3. Complications of Idiopathic Nephrotic Syndrome and Drug Side Effects in Patients Under Study & Number(\%) \\
\hline Variable & $17(5.7)$ \\
\hline Side Effects of the Disease & $28(9.4)$ \\
\hline Infection & $3(1.0)$ \\
\hline High blood pressure & $4(1.3)$ \\
\hline Acute renal failure (Hypovolemic) & $0(0.0)$ \\
\hline Anemia & $0(0.0)$ \\
\hline Thromboembolism & \\
\hline Leukopenia & $23(7.7)$ \\
\hline Drug Side Effects & $8(2.7)$ \\
\hline Cataract & $1(0.3)$ \\
\hline Cushingoid face & $0(0)$ \\
\hline High blood pressure & $2(0.7)$ \\
\hline Leukopenia & $7(2.3)$ \\
\hline Increased liver enzymes & \\
\hline Renal impairment &
\end{tabular}

\begin{tabular}{|c|c|c|c|c|c|c|}
\hline \multirow[t]{2}{*}{ Variable } & \multicolumn{5}{|c|}{ Patients' Age (Years) } & \multirow[t]{2}{*}{ PValue $^{\mathrm{a}}$} \\
\hline & $1-2$ & 2-4 & 4-6 & 6-8 & $>8$ & \\
\hline Type of nephrotic syndrome & & & & & & 0.007 \\
\hline Resistant to treatment & $5(12.5)$ & $9(7.5)$ & $5(7.4)$ & $2(6.3)$ & $12(30.8)$ & \\
\hline Steroid dependent/frequent relapse & $26(65.0)$ & $57(47.5)$ & $35(51.5)$ & $18(56.3)$ & $15(38.5)$ & \\
\hline Infrequent recurrence & $5(12.5)$ & $15(12.5)$ & $8(11.8)$ & $2(6.3)$ & $3(7.7)$ & \\
\hline No recurrence & $4(10.0)$ & $39(32.5)$ & $20(29.4)$ & $10(31.3)$ & $9(23.1)$ & \\
\hline Number of recurrences during the follow-up & & & & & & 0.406 \\
\hline No recurrence & $4(11.4)$ & $39(35.1)$ & $20(31.7)$ & $10(33.3)$ & $9(33.3)$ & \\
\hline 1 & $11(31.4)$ & $28(25.2)$ & $11(17.5)$ & $8(26.7)$ & $9(33.3)$ & \\
\hline 2 & $9(25.7)$ & $15(13.5)$ & $18(28.6)$ & $6(20.0)$ & $5(18.5)$ & \\
\hline 3 & $6(17.1)$ & $10(9.0)$ & $5(7.9)$ & $3(10.0)$ & $3(11.1)$ & \\
\hline 4 & $3(8.6)$ & $11(9.9)$ & $3(4.8)$ & $1(3.3)$ & $0(0.0)$ & \\
\hline$\geq 5$ & $2(5.7)$ & $8(7.2)$ & $6(9.5)$ & $2(6.7)$ & $1(3.7)$ & \\
\hline Annual recurrence rate during the follow-up & & & & & & 0.332 \\
\hline None & $4(11.4)$ & $39(35.1)$ & $20(31.7)$ & $10(33.3)$ & $9(33.3)$ & \\
\hline $0.01-1.00$ & $26(74.3)$ & $57(51.4)$ & $35(55.6)$ & $20(66.7)$ & $16(59.3)$ & \\
\hline $1.01-2$ & $4(11.4)$ & $13(11.7)$ & $6(9.5)$ & $0(0.0)$ & $2(7.4)$ & \\
\hline$\geq 2$ & $1(2.9)$ & $2(1.8)$ & $2(3.2)$ & $0(0.0)$ & $0(0.0)$ & \\
\hline
\end{tabular}

${ }^{\mathrm{a}}$ Chi-square Test. 


\begin{tabular}{|c|c|c|c|}
\hline \multirow[t]{2}{*}{ Variable } & \multicolumn{2}{|c|}{ Sex } & \multirow[t]{2}{*}{ PValue $^{a}$} \\
\hline & Boy & Girl & \\
\hline Type of nephrotic syndrome & & & 0.181 \\
\hline Resistant to treatment & $15(8.1)$ & $18(15.8)$ & \\
\hline Infrequent recurrence & $19(10.3)$ & $14(12.3)$ & \\
\hline No recurrence & $53(28.6)$ & $29(25.4)$ & \\
\hline Number of recurrences during the follow-up & & & 0.922 \\
\hline No recurrence & $53(31.2)$ & $29(30.2)$ & \\
\hline 1 & $46(27.1)$ & $21(21.9)$ & \\
\hline 2 & $31(18.2)$ & $22(22.9)$ & \\
\hline 3 & $17(10.0)$ & $10(10.4)$ & \\
\hline 4 & $11(6.5)$ & $7(7.3)$ & \\
\hline$\geq 5$ & $12(7.1)$ & $7(7.3)$ & \\
\hline Annual recurrence rate during the follow-up & & & 0.684 \\
\hline None & $53(31.2)$ & $29(30.2)$ & \\
\hline $0.01-1.00$ & $100(58.8)$ & $54(56.3)$ & \\
\hline $1.01-2$ & $15(8.8)$ & $10(10.4)$ & \\
\hline$\geq 2$ & $2(1.2)$ & $3(3.1)$ & \\
\hline
\end{tabular}

${ }^{\mathrm{a}}$ Chi-square Test.

tients recovered with early steroid treatment. In these patients, recurrences are common, and a significant number of them experience steroid dependence or frequent relapse. Therefore, their long-term follow-up is necessary (5).

Another study in India showed that the early quarterly treatment with corticosteroids is the most important determinant of prognosis in response to steroids in children. Moreover, more than $70 \%$ of children had a relapse with steroid-sensitive nephrotic syndrome, and almost $50 \%$ had recurrent relapse or steroid-dependence, which is highly similar to our findings. Besides, $15-25 \%$ of the patients experienced relapses within 10 to 15 years after the onset of nephrotic syndrome. Furthermore, the age of onset and the frequency of recurrence in childhood were associated with recurrence in adulthood (19). Ozlu et al. indicated that the male gender, age older than eight years at the onset of the disease, and the presence of microscopic hematuria were factors that predicted the lack of response to steroids (20). However, the current study showed that advanced age is the sole predictor of steroid resistance. In the research conducted by Samiulus et al., 80.4\% of patients responded to steroids. The renal biopsy results showed that the diseases with minimal changes and mesangial proliferation were 20.60 and $21.90 \%$, respectively. Response to steroids was higher in children younger than five years, corroborating our results on the relationship between age and response to steroids. Steroid resistance was higher in children with Focal Segmental Glomerulonephritis (FSGN). Furthermore, complete remission was observed in $96 \%$ of patients who showed steroid sensitivity and $46.6 \%$ who were steroid-resistant. Fifteen percent of steroid-resistant patients developed renal failure. Intermittent infections and response to steroid therapy were important factors in the prognosis of nephrotic syndrome (21).

In the present study, nephrotic syndrome with minimal changes was the most common pathological finding in steroid-resistant patients. However, in other studies, the most common pathological finding was Focal Segmental Glomerulonephritis (FSGN) (20,22). This difference may be due to the biopsy time, skill, and experience of the pathologist, sampling method, small number of patients who underwent biopsy, and patients who refused to experience biopsy.

\subsection{Strengths and Limitations}

The present study has some strengths and limitations. The most considerable strength of this cross-sectional study is its population-based nature with a large number 
of patients. In addition, this research was performed during a 20-year period. The most important limitation of the study is its cross-sectional design. Second, the information may not have been accurately recorded due to the retrospective nature of the study; for example, there were not accurate data about physical growth in patient files.

\subsection{Conclusion}

This study revealed that clinical presentations of INS and patient age and sex distribution were similar to other national and international reports. Higher age at the time of diagnosis was significantly associated with steroid resistance. However, there was no significant relationship between age at the time of diagnosis and recurrence of the disease. On the other hand, there was no significant relationship between gender and steroid response or disease recurrence. The interval between response to treatment and first recurrence had not any influence on the recurrence rate.

\section{Acknowledgments}

We thank the honorable Research Vice-Chancellor of the University and the Faculty of Medicine of Kermanshah University of Medical Sciences.

\section{Footnotes}

Authors' Contribution: AS and MT designed the study, observed the accuracy and validity of the study, and supervised the project. SS and MS collected the data and conducted the follow-up study. SA did the statistical analysis of the data and interpreted the results. AS and MT wrote the paper. All authors edited and revised the final manuscript and agreed with its publication.

Conflict of Interests: The authors have no conflicts of interest to disclose.

Ethical Approval: The study was approved by the Ethics Committee of Kurdistan University of Medical Sciences (Ethical Number: IR.MUK.REC.1398.741).

Funding/Support: This study was supported in part by grant CAR01988 from the National University of Health and by a teaching and research scholarship from the Kermanshah College of Medicine (Dr. Abolhassan Seyezadeh).

\section{References}

1. Hakimi A, Valavi E, Madhushimazrae S, Latifi M, Dashtbozorge B. The effect of continuous care model on parents' knowledge and controlling symptoms and recurrence in children with nephrotic syndrome. Journal of Clinical Nursing and Midwifery. 2016;5(2).
2. Yousefichaijan P, Rezagholizamenjany M, Rafiei F, Taherahmadi H, Rafiei M. The relationship between blood biomarkers level and the prognosis of nephrotic syndrome in the children. International Journal of Pediatrics. 2016;4(9):3489-97.

3. Chen YM, Miner JH. Glomerular basement membrane and related glomerular disease. Transl Res. 2012;160(4):291-7. doi: 10.1016/j.trsl.2012.03.004. [PubMed: 22683419]. [PubMed Central: PMC3477400].

4. Behrman A, Kliegman K, Jenson L. Nelson text book of pediatrics. 19th ed ed. Saunders: Elsevier; 2011.

5. Seyedzadeh A, Alimohammadi E, Soleimani A. Clinical Feature of Idiopathic Nephrotic Syndrome In Children Referring To Pediatric Nephrology Clinic During1380-1390 Kermanshah. The Journal of Urmia University of Medical Sciences. 2014;24(11):927-32.

6. Eddy AA, Symons JM. Nephrotic syndrome in childhood. The Lancet. 2003;362(9384):629-39. doi: 10.1016/s0140-6736(03)14184-0.

7. Beck L, Bomback AS, Choi MJ, Holzman LB, Langford C, Mariani LH, et al. KDOQI US commentary on the 2012 KDIGO clinical practice guideline for glomerulonephritis. Am J Kidney Dis. 2013;62(3):403-41. doi: 10.1053/j.ajkd.2013.06.002. [PubMed: 23871408].

8. Pokrajac D, Kamber AH, Karasalihovic Z. Children with SteroidResistant Nephrotic Syndrome: A Single -Center Experience. Mater Sociomed. 2018;30(2):84-8. doi: 10.5455/msm.2018.30.84-88. [PubMed: 30061794]. [PubMed Central: PMC6029918].

9. Kyrieleis HA, Lowik MM, Pronk I, Cruysberg HR, Kremer JA, Oyen WJ, et al. Long-term outcome of biopsy-proven, frequently relapsing minimal-change nephrotic syndrome in children. Clin J Am Soc Nephrol. 2009;4(10):1593-600. doi: 10.2215/CJN.05691108. [PubMed: 19808243]. [PubMed Central: PMC2758253].

10. Filler G. Treatment of nephrotic syndrome in children and controlled trials. Nephrol Dial Transplant. 2003;18 Suppl 6:vi75-8. doi: 10.1093/ndt/gfg1059. [PubMed: 12953047].

11. Kallash M, Smoyer WE, Mahan JD. Rituximab Use in the Management of Childhood Nephrotic Syndrome. Front Pediatr. 2019;7:178. doi: 10.3389/fped.2019.00178. [PubMed: 31134169]. [PubMed Central: PMC6524616].

12. Noone DG, Iijima K, Parekh R. Idiopathic nephrotic syndrome in children. The Lancet. 2018;392(10141):61-74. doi: 10.1016/s01406736(18)30536-1.

13. Seyedzadeh A, Tohidi MR, Amiri R, Farshchian N, Golpayegany MR, Hookari S, et al. Relationship of Renal Cortical Echogenicity in Renal Ultrasonography with Course of Disease in Children with Idiopathic Nephrotic Syndrome. International Journal of Pediatrics. 2020;8(8):11673-80.

14. Kawasaki Y, Suzuki J, Nozawa R, Suzuki S, Suzuki H. Prediction of relapse by plasma lipoprotein(a) concentration in children with steroid-sensitive nephrotic syndrome. Nephron. 2002;92(4):807-11. doi: 10.1159/000065452. [PubMed: 12399624].

15. Rezavand N, Seyedzadeh A, Tohidi M, Seyedzadeh M, Hookary S, Abdi A. The relationship between low-birth weight and nephrotic syndrome in children. Journal of Nephropharmacology. 2017;7(1):6-9. doi: 10.15171/npj.2018.03.

16. Niaudet P, Boyer O. Idiopathic Nephrotic Syndrome in Children: Clinical Aspects. Pediatric Nephrology. USA: Springer; 2016. p. 839-82. doi: 10.1007/978-3-662-43596-0_24.

17. Ahmadzadeh A, Derakhshan A, Hakimzadeh M, Zolfigol A. Idiopathic nephrotic syndrome in Iranian children. Indian pediatrics. 2008;45(1):52

18. Wong W. Idiopathic nephrotic syndrome in New Zealand children, demographic, clinical features, initial management and outcome after twelve-month follow-up: results of a three-year national surveillance study.JPaediatr Child Health.2007;43(5):337-41. doi:10.1111/j.14401754.2007.01077.x. [PubMed: 17489822].

19. Bagga A, Mantan M. Nephrotic syndrome in children. Indian Journal of medical research. 2005;122(1):13. 
20. Mortazavi F. [Idiopathic nephrotic syndrome: Clinicopathological study in Tabriz Children Hospital]. Urmia Med J. 2006;18(4):304-9.

21. Ozlu SG, Demircin G, Tokmeci N, Caltik Yilmaz A, Aydog O, Bulbul M, et al. Long-term prognosis of idiopathic nephrotic syndrome in children. Ren Fail. 2015;37(4):672-7. doi: 10.3109/0886022X.2015.1010940.
[PubMed: 25687382].

22. Safaei A, Maleknejad S. Spectrum of childhood nephrotic syndrome in Iran: A single center study. Indian J Nephrol. 2009;19(3):87-90. doi: 10.4103/0971-4065.57103. [PubMed: 20436726]. [PubMed Central: PMC2859484]. 